\title{
Adaptive Blind Equalization of QAM Transmitted Constellations across Linear Band-Limited Channel
}

\author{
Ram Nishanth Vanka ${ }^{1}$, S Balarama Murty ${ }^{2}$, B Chandra Mouli ${ }^{3}$ \\ ${ }^{1}$ M.Tech (Communication Systems) \\ ${ }^{2}$ (Department of ECE, Anil Neerukonda Institute of Technology and Sciences, Vizag, Andhra Pradesh, India) \\ ${ }^{3}$ (Department of ECE, Anil Neerukonda Institute of Technology and Sciences, Vizag, Andhra Pradesh, India)
}

\begin{abstract}
ISI (Intersymbol Interference) severely degrades the performance of high speed digital communication systems. A popular technique for countering ISI is Adaptive Equalization. Blind/Unsupervized equalization is one class of adaptive equalization where initial training of equalizer's weights is not feasible. Godard's Constant Modulus Algorithm (CMA) was the very first blind equalization algorithm that could be applied for two dimensional communication systems. It requires the transmitted signal's statistics to perform equalization. Picchi \& Prati modified the standard decision-directed algorithm and proposed a new algorithm by the name Stop-and-Go. Wei Rao by simply converting the equalizer's output proposed a modified version of the CMA. This paper compares the performance of blind equalization algorithms: CMA, Stop-and-Go decisiondirected algorithm and Wei Rao's modified constant modulus algorithm for 16 and 64-QAM constellations transmitted across a linear band-limited channel. Simulation results indicate impulse response of the cascade of transmission channel and equalizer (after convergence) is similar to that of ideal transmission channel's impulse response. The results also show that stop-and-go outperforms the rest in terms of convergence rate and MSE.
\end{abstract}

Keywords: Adaptive Equalization, Blind Equalization, CMA, ISI, Stop-and-Go

\section{Introduction}

The presence of ISI (Intersymbol Interference) in digital communications is an undisputed fact. Non ideal band-limited channels distort the transmitted signal in both amplitude and phase; because of this pulses transmitted in succession at rates comparable to the bandwidth are smeared to the point that they are no longer distinguishable as well defined pulses at the receiving terminal [1]. Instead they overlap giving rise to ISI. ISI makes high speed communication unreliable by causing high error rates at the receiver. Equalization is defined as any signal processing technique that can counter ISI. In practice, the characteristics of transmission channels are unknown a priori and most of the channels are time-variant. Because of this, equalizers that are adaptive to the variations in the channels response are designed [1]. Adaptive Equalization can be classified into Supervized/Trained and Blind/Unsupervized. In supervized equalization a particular training sequence that is available both at the transmitter and receiver in proper synchronism is transmitted for the purpose of initial training of equalizer's weights [2]. On the contrary initial training of the weights cannot be done in Blind/Unsupervized as transmission of training sequence is not possible.

Sato was the first to propose an algorithm for performing blind equalization [3]. His algorithm was proposed in the context of binary pulse amplitude modulated signals. The significance of blind equalizers was well explained by Godard [4]. Godard proposed Constant Modulus Algorithm (CMA) and it is the first blind algorithm that could be applied for two dimensional communication systems. CMA requires the transmitted signal's statistics in order to perform equalization. Picchi and Prati's [5] Stop-and-Go Decision-Directed algorithm is basically a modified version of the standard decision-directed algorithm. The modification is made in the weight updation process with the help of a binary flag. This binary flag dictates whether the weight updation should be done or not. By simple adjustment of the equalizer output, Wei Rao [6] proposed a modified version of CMA. Unlike CMA, Wei Rao's modified CMA requires only the statistics of 4-QAM (Quadrature Amplitude Modulation) signal regardless of the transmitted signal. The basic aim of all the three algorithms is to make the impulse response of the cascade of the transmission channel and the equalizer (after convergence) to match the impulse response of the ideal transmission channel. Impulse response of an ideal transmission channel is given by:

$$
\mathrm{h}(\mathrm{k})=\mathrm{A} \delta(\mathrm{k}-\mathrm{n})
$$

where $\mathrm{k}$ denotes the time instant, $\mathrm{n}$ denotes the propagation delay and $\delta(\mathrm{k})$ is the Dirac delta function (Unit Impulse Function). In this paper performance of the three algorithms (CMA, Stop-and-Go and Wei Rao's modified CMA) is compared in terms of their convergence rate to investigate which blind algorithm has quicker learning capacity for 16 and 64-QAM transmitted constellations across a linear band-limited channel. 
This paper is organized as follows: Sections II, III and IV introduce the idea behind CMA, Stop-andGo and Wei Rao's modified CMA. The simulation results are available in section V and section VI gives the conclusion.

\section{Constant Modulus Algorithm (CMA)}

Godard proposed a nonconvex Mean Square Error (MSE) cost function given by:

$$
\mathrm{J}_{\mathrm{CMA}}(\mathrm{k})=\mathrm{E}\left[\left\{|\mathrm{y}(\mathrm{k})|^{2}-\mathrm{R}_{2}\right\}^{2}\right]
$$

where $y(k)$ is the equalizer's instantaneous output and $\mathrm{R}_{2}$ is called constant modulus. The N-tap weighted equalizer's output is given by:

$$
\mathrm{y}(\mathrm{k})=\mathrm{w}(\mathrm{k})^{\mathrm{H}} \mathrm{x}(\mathrm{k})
$$

$\mathrm{H}$ represents Hermitian operation (conjugate transpose), $\mathrm{x}(\mathrm{k})=[x(\mathrm{k}), x(\mathrm{k}-1), \ldots, x(\mathrm{k}-\mathrm{N}+1)]^{\mathrm{T}}$ is the equalizer's instantaneous input vector and $\mathrm{w}(\mathrm{k})=\left[w_{0}(\mathrm{k}), w_{1}(\mathrm{k}), \ldots, w_{N-1}(\mathrm{k})\right]^{\mathrm{T}}$ is the equalizer's instantaneous weight vector where $\mathrm{T}$ represents transpose operation. The constant modulus is defined as:

$$
\mathrm{R}_{2}=\frac{\mathrm{E}\left[\left.\mathrm{a}(\mathrm{k})\right|^{4}\right]}{\mathrm{E}\left[\left.\mathrm{a}(\mathrm{k})\right|^{2}\right]}
$$

$\mathrm{a}(\mathrm{k})$ is the instantaneous transmitted symbol. CMA is designed to penalize deviations of the blind equalizer output $\mathrm{y}(\mathrm{k})$ from the constant modulus [7]. The tap weight vector of the equalizer is adapted in accordance with the stochastic gradient algorithm [4]

$$
\mathrm{w}(\mathrm{k}+1)=\mathrm{w}(\mathrm{k})-\alpha_{\mathrm{CMA}} \mathrm{x}(\mathrm{k}) \mathrm{e}_{\mathrm{CMA}}(\mathrm{k})^{*}
$$

$\alpha$ is a real valued parameter called the adaptation step size and $e_{\mathrm{CMA}}(\mathrm{k})$ is the instantaneous error signal given by:

$$
\mathrm{e}_{\mathrm{CMA}}(\mathrm{k})=\mathrm{y}(\mathrm{k})\left[\mathrm{y}(\mathrm{k}) \mid-\frac{\mathrm{R}_{2}}{|\mathrm{y}(\mathrm{k})|}\right]
$$

* represents conjugate operation. In Fig.1 CMA mode of operation can be obtained by keeping the switch at position1.

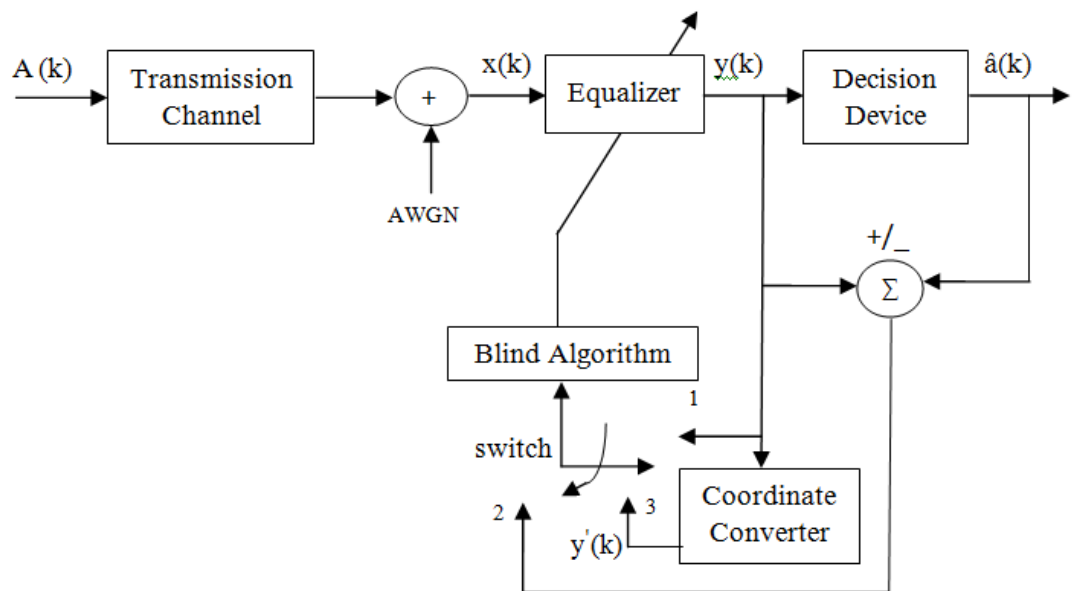

Fig. 1 Communication System with a Blind Adaptive Equalizer

\section{Stop-and-Go Decision-Directed Algorithm}

The goal of Picchi and Prati in [5] proposing Stop-and-Go algorithm was to modify the standard decision-directed (DD) algorithm [8] such that it retains it's simplicity and improves blind convergence capabilities. Picchi and Prati employed a binary valued flag $f(k)$ which dictates whether the weight adaptation should be carried on or not. The weight updation is carried on if the self decided output error on the current decision is reliable to be used in the DD algorithm, if it is not, then the updation is stopped for that iteration. The MSE cost function is defined as:

$$
\mathrm{J}_{\mathrm{SG}}=\mathrm{E}\left[\hat{\mathrm{e}}^{\mathrm{SG}}(\mathrm{k})\right]^{2}
$$

where $\hat{\mathrm{e}}^{\mathrm{sG}}(\mathrm{k})$ the self decided error is defined as:

$$
\hat{e}^{S G}(k)=y(k)-\hat{a}(k)
$$

The weight update equation is given as: 


$$
\begin{aligned}
& \mathrm{w}_{\mathrm{r}}^{\mathrm{SG}}(\mathrm{k}+1)=\mathrm{w}_{\mathrm{r}} \mathrm{SG}^{\mathrm{S}}(\mathrm{k})-\alpha_{\mathrm{SG}}\left[\mathrm{f}_{\mathrm{r}}(\mathrm{k}) \hat{\mathrm{e}}_{\mathrm{r}}^{\mathrm{SG}}(\mathrm{k}) \mathrm{x}_{\mathrm{r}}(\mathrm{k})+\mathrm{f}_{\mathrm{i}}(\mathrm{k}) \hat{\mathrm{e}}_{\mathrm{i}}^{\mathrm{SG}}(\mathrm{k}) \mathrm{x}_{\mathrm{i}}(\mathrm{k})\right] \\
& \mathrm{w}_{\mathrm{i}}^{\mathrm{SG}}(\mathrm{k}+1)=\mathrm{w}_{\mathrm{i}}^{\mathrm{SG}}(\mathrm{k})-\alpha_{\mathrm{SG}}\left[\mathrm{f}_{\mathrm{r}}(\mathrm{k}) \hat{e}_{\mathrm{r}}^{\mathrm{SG}}(\mathrm{k}) \mathrm{x}_{\mathrm{i}}(\mathrm{k})-\mathrm{f}_{\mathrm{i}}(\mathrm{k}) \hat{\mathrm{e}}_{\mathrm{i}}^{\mathrm{SG}}(\mathrm{k}) \mathrm{x}_{\mathrm{r}}(\mathrm{k})\right]
\end{aligned}
$$

subscripts $r$ and $i$ indicate real and imaginary parts of the corresponding quantities. The generation of the binary flag $\mathrm{f}(\mathrm{k})$ depends on two Sato [3] like errors given by:

$$
\begin{aligned}
& \tilde{\mathrm{e}}_{\mathrm{r}}(\mathrm{k})=\mathrm{y}_{\mathrm{r}}(\mathrm{k})-\beta\left(\operatorname{sgn}\left(\mathrm{y}_{\mathrm{r}}(\mathrm{k})\right)\right) \\
& \tilde{\mathrm{e}}_{\mathrm{i}}(\mathrm{k})=\mathrm{y}_{\mathrm{i}}(\mathrm{k})-\beta\left(\operatorname{sgn}\left(\mathrm{y}_{\mathrm{i}}(\mathrm{k})\right)\right)
\end{aligned}
$$

where $\beta$ is a real value which is chosen in accordance with the transmitted constellation and sgn represents the sign of the variable under consideration. The binary flag generation is accomplished in the following manner:

$$
\begin{aligned}
& \mathrm{f}_{\mathrm{r}}(\mathrm{k})=\left\{\begin{array}{ll}
1, \text { if } & \hat{\mathrm{e}}_{\mathrm{r}}^{\mathrm{SG}}=\tilde{\mathrm{e}}_{\mathrm{r}}(\mathrm{k}) \\
0, \text { if } & \hat{\mathrm{e}}_{\mathrm{r}}^{\mathrm{SG}} \tilde{\mathrm{e}}_{\mathrm{r}}(\mathrm{k})
\end{array}\right\} \\
& \mathrm{f}_{\mathrm{i}}(\mathrm{k})=\left\{\begin{array}{ll}
1, \text { if } & \hat{\mathrm{e}}_{\mathrm{i}}^{\mathrm{SG}}=\tilde{\mathrm{e}}_{\mathrm{i}}(\mathrm{k}) \\
0, \text { if } & \hat{\mathrm{e}}_{\mathrm{i}}^{\mathrm{SG}} \neq \tilde{\mathrm{e}}_{\mathrm{i}}(\mathrm{k})
\end{array}\right\}
\end{aligned}
$$

In Fig.1 Stop-and-Go mode of operation can be obtained by keeping the switch at position 2 .

\section{Wei Rao's Modified CMA}

Wei Rao and his team of researchers proposed a new CMA cost function which performs equalization of higher order QAM input signals with the help of 4-QAM signal statistics. In addition, order detection of transmitted QAM signal is also achieved. Wei Rao proposed this new CMA algorithm by exploiting the inherent structural relationship between the 4-QAM signal coordinates and other higher order QAM signal coordinates [6].Wei Rao's cost function is defined as:

$$
\mathrm{J}_{\mathrm{CMAm}}(\mathrm{k})=\mathrm{J}_{\mathrm{CMAmr}}(\mathrm{k})+\mathrm{j} \mathrm{J}_{\mathrm{CMAmi}}(\mathrm{k})
$$

where

$$
\begin{aligned}
\mathrm{J}_{\mathrm{CMAMI}}(\mathrm{k}) & =\mathrm{E}\left[\left(\left|\mathrm{y}^{\prime}(\mathrm{k})\right|^{2}-\mathrm{R}_{2}{ }^{4 \mathrm{r}}\right)^{2}\right] \\
\mathrm{J}_{\mathrm{CMAmi}}(\mathrm{k}) & =\mathrm{E}\left[\left(\left|\mathrm{y}^{\prime}(\mathrm{k})\right|^{2}-\mathrm{R}_{2}{ }^{4 \mathrm{i}}\right)^{2}\right]
\end{aligned}
$$

$\mathrm{R}_{2}{ }^{4 \mathrm{r}}$ and $\mathrm{R}_{2}{ }^{4 \mathrm{i}}$ represent the constant modulus of the real and imaginary parts of the 4-QAM signal. For 4-QAM signal

$$
\mathrm{R}_{2}{ }^{4 \mathrm{r}}=\mathrm{R}_{2}{ }^{4 \mathrm{i}}=1
$$

$y^{\prime}(k)$ is obtained by converting the coordinates of the equalizer output $y(k)$. $y^{\prime}(k)$ is defined as

$$
\begin{aligned}
& \mathrm{y}_{\mathrm{r}}^{\prime}(\mathrm{k})=\mathrm{y}_{\mathrm{r}}(\mathrm{k})-4 \operatorname{sign}\left(\mathrm{y}_{\mathrm{r}}(\mathrm{k})\right)-2 \operatorname{sgn}\left(\mathrm{y}_{\mathrm{r}}(\mathrm{k})-4 \operatorname{sign}\left(\mathrm{y}_{\mathrm{r}}(\mathrm{k})\right)\right) \\
& \mathrm{y}_{\mathrm{i}}^{\prime}(\mathrm{k})=\mathrm{y}_{\mathrm{i}}(\mathrm{k})-4 \operatorname{sign}\left(\mathrm{y}_{\mathrm{i}}(\mathrm{k})\right)-2 \operatorname{sgn}\left(\mathrm{y}_{\mathrm{i}}(\mathrm{k})-4 \operatorname{sign}\left(\mathrm{y}_{\mathrm{i}}(\mathrm{k})\right)\right)
\end{aligned}
$$

where sgn represents the signum function. The above equation is applicable for changing the coordinates of 16QAM, 32-QAM and 64-QAM constellation points to that of 4-QAM constellation points [6]. By using this coordinate conversion technique Wei Rao was able to modify CMA. The result of applying (15) to 64-QAM is 4-QAM, and it is shown in Table I. In Fig.1 Wei Rao's modified CMA mode of operation can be obtained by keeping the switch at position 3 .

Table I: Conversion of 64-QAM coordinates to 4-QAM coordinates

\begin{tabular}{|c|c|c|c|}
\hline $\begin{array}{c}\text { Actual 64-QAM } \\
\text { Coordinates }\end{array}$ & Modified Coordinates & $\begin{array}{c}\text { Actual 64-QAM } \\
\text { Coordinates }\end{array}$ & Modified Coordinates \\
\hline$-7+7 \mathrm{j}$ & $-1+1 \mathrm{j}$ & $7+7 \mathrm{j}$ & $1+1 \mathrm{j}$ \\
\hline$-7+5 \mathrm{j}$ & $-1-1 \mathrm{j}$ & $7+5 \mathrm{j}$ & $1-1 \mathrm{j}$ \\
\hline$-7+3 \mathrm{j}$ & $-1+1 \mathrm{j}$ & $7+3 \mathrm{j}$ & $1+1 \mathrm{j}$ \\
\hline$-7+1 \mathrm{j}$ & $-1-1 \mathrm{j}$ & $7+1 \mathrm{j}$ & $1-1 \mathrm{j}$ \\
\hline$-7-1 \mathrm{j}$ & $-1+1 \mathrm{j}$ & $7-1 \mathrm{j}$ & $1+1 \mathrm{j}$ \\
\hline
\end{tabular}




\begin{tabular}{|c|c|c|c|}
\hline$-7-3 j$ & $-1-1 \mathrm{j}$ & $7-3 j$ & $1-1 j$ \\
\hline$-7-5 j$ & $-1+1 j$ & $7-5 j$ & $1+1 \mathrm{j}$ \\
\hline$-7-7 j$ & $-1-1 \mathrm{j}$ & $7-7 j$ & $1-1 \mathrm{j}$ \\
\hline$-5+7 j$ & $1+1 \mathrm{j}$ & $5+7 \mathrm{j}$ & $-1+1 \mathrm{j}$ \\
\hline$-5+3 j$ & $1+1 \mathrm{j}$ & $5+3 \mathrm{j}$ & $-1+1 j$ \\
\hline$-5+1 j$ & $1-1 \mathrm{j}$ & $5+1 \mathrm{j}$ & $-1-1 \mathrm{j}$ \\
\hline$-5-1 j$ & $1+1 \mathrm{j}$ & $5-1 \mathrm{j}$ & $-1+1 j$ \\
\hline$-5-5 j$ & $1+1 \mathrm{j}$ & $5-5 j$ & $-1+1 j$ \\
\hline$-5-7 j$ & $1-1 \mathrm{j}$ & $5-7 \mathrm{j}$ & $-1-1 \mathrm{j}$ \\
\hline$-3+7 j$ & $-1+1 j$ & $3+7 \mathrm{j}$ & $1+1 \mathrm{j}$ \\
\hline$-3+5 j$ & $-1-1 \mathrm{j}$ & $3+5 \mathrm{j}$ & $1-1 \mathrm{j}$ \\
\hline$-3+3 j$ & $-1+1 j$ & $3+3 \mathrm{j}$ & $1+1 j$ \\
\hline$-3+1 j$ & $-1-1 j$ & $3+1 \mathrm{j}$ & $1-1 \mathrm{j}$ \\
\hline$-3-7 j$ & $-1-1 j$ & $3-7 j$ & $1-1 \mathrm{j}$ \\
\hline$-1+7 j$ & $1+1 \mathrm{j}$ & $1+7 \mathrm{j}$ & $-1+1 \mathrm{j}$ \\
\hline$-1+5 j$ & $1-1 \mathrm{j}$ & $1+5 \mathrm{j}$ & $-1-1 \mathrm{j}$ \\
\hline$-1+3 j$ & $1+1 \mathrm{j}$ & $1+3 \mathrm{j}$ & $-1+1 \mathrm{j}$ \\
\hline$-1+1 j$ & $1-1 j$ & $1+1 \mathrm{j}$ & $-1-1 j$ \\
\hline$-1-1 j$ & $1+1 \mathrm{j}$ & $1-1 \mathrm{j}$ & $-1+1 \mathrm{j}$ \\
\hline$-1-3 j$ & $1-1 j$ & $1-3 j$ & $-1-1 j$ \\
\hline$-1-5 j$ & $1+1 \mathrm{j}$ & $1-5 j$ & $-1+1 j$ \\
\hline$-1-7 \mathrm{j}$ & $1-1 j$ & $1-7 j$ & $-1-1 \mathrm{j}$ \\
\hline
\end{tabular}

The weight update equation is given by

$$
\hat{\mathbf{w}}(\mathrm{k}+1)=\hat{\mathbf{w}}(\mathrm{k})-\mu_{\mathrm{CMAm}} \mathrm{x}(\mathrm{k})\left[\mathrm{e}_{\mathrm{r}}(\mathrm{k})+\mathrm{je}_{\mathrm{i}}(\mathrm{k})\right]^{*}
$$

where

$$
\begin{aligned}
& \mathrm{e}_{\mathrm{r}}(\mathrm{k})=\left[\left|\mathrm{y}_{\mathrm{r}}^{\prime}(\mathrm{k})\right|^{2}-\mathrm{R}_{2}^{4 \mathrm{r}}\right] \mathrm{y}_{\mathrm{r}}^{\prime}(\mathrm{k}) \\
& \mathrm{e}_{\mathrm{i}}(\mathrm{k})=\left[\left|\mathrm{y}_{\mathrm{i}}^{\prime}(\mathrm{k})\right|^{2}-\mathrm{R}_{2}^{4 \mathrm{i}}\right] \mathrm{y}_{\mathrm{i}}^{\prime}(\mathrm{k})
\end{aligned}
$$

\section{Simulation Results}

Simulation is done for 16 and 64 rectangular Quadrature Amplitude Modulated (QAM) transmitted constellations for the linear band-limited transmission channel 1 considered in [4]. A 21-tap feed forward equalizer with SNR of $30 \mathrm{~dB}$ is considered for all the three algorithms. Initial tap weight settings correspond to closed eye condition. From Fig. 2 to Fig. 4, impulse response of the cascade of transmission channel and the equalizer (after convergence) for 16-QAM transmission is presented for all the three algorithms considered. From Fig. 5 to Fig. 7, impulse response for 64-QAM transmission is presented for all the three algorithms considered. The Learning Curves of CMA, Stop-and-Go and Wei Rao's modified CMA are shown for 16-QAM in Fig. 8 and in Fig. 9 for 64-QAM.

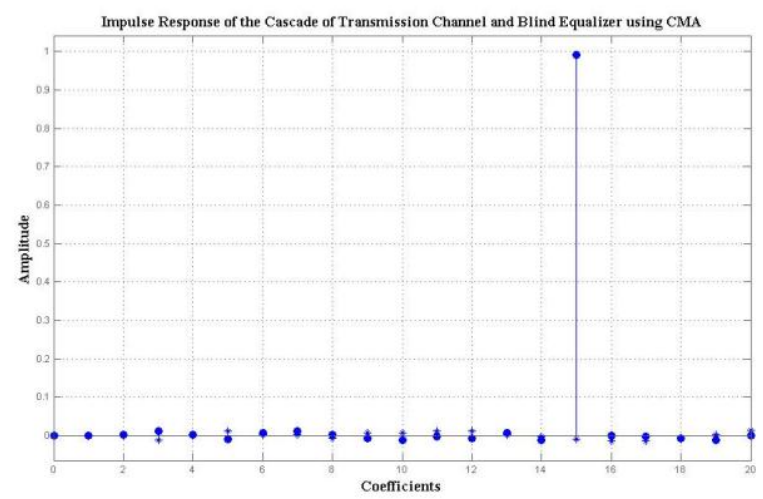




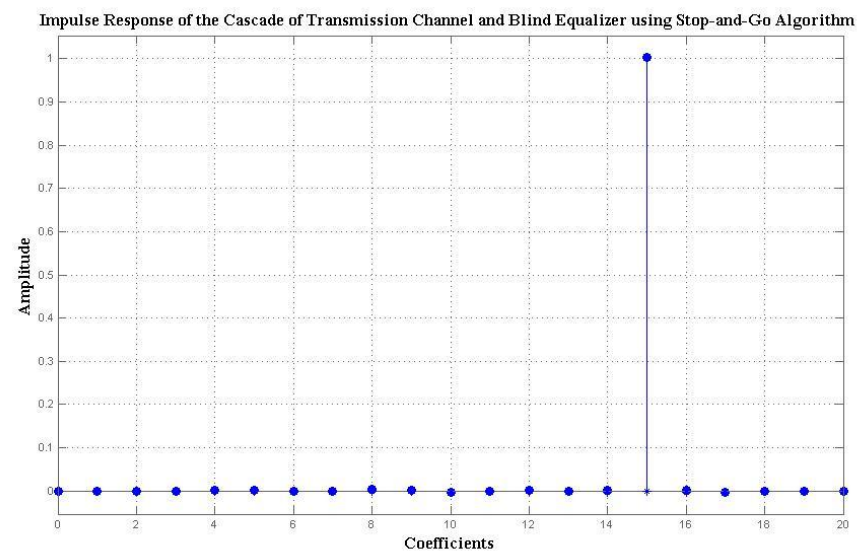

Fig.3 Impulse response of the cascade of transmission channel and the Stop-and-Go blind equalizer for 16QAM transmission

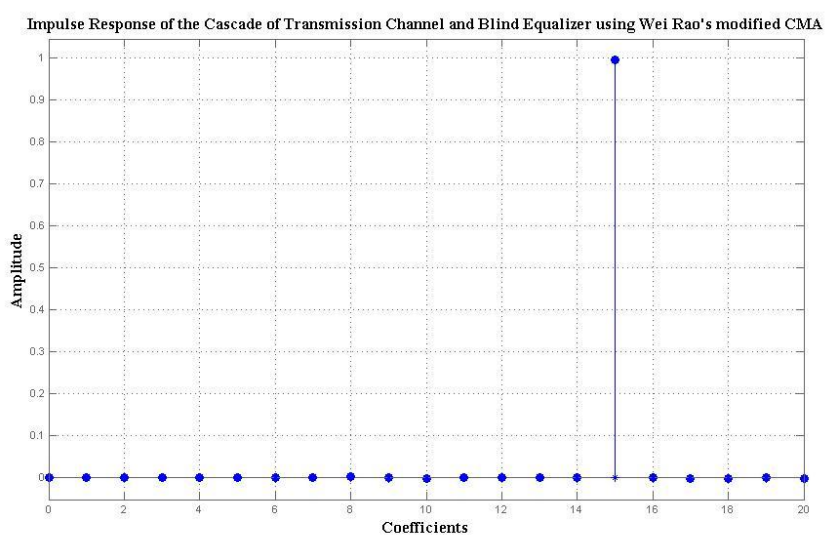

Fig.4 Impulse response of the cascade of transmission channel and the Wei Rao's modified CMA blind equalizer for 16-QAM transmission

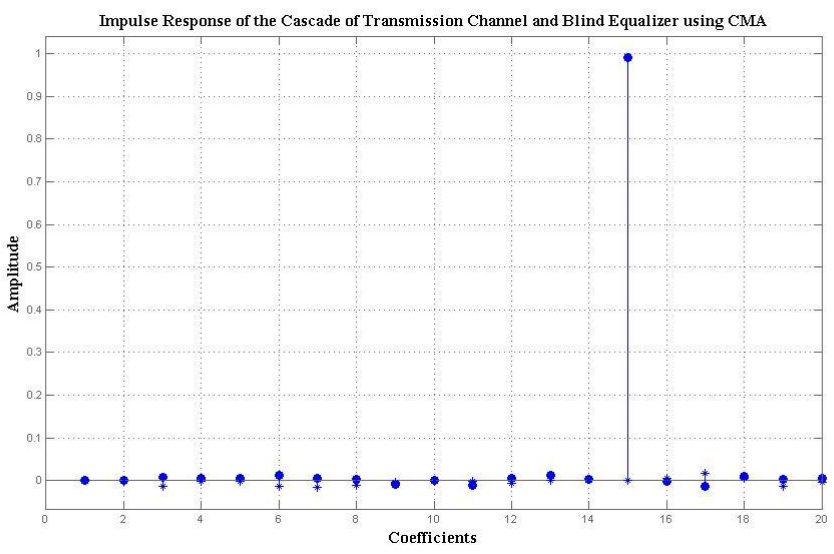

Fig.5 Impulse response of the cascade of transmission channel and the CMA blind equalizer for 64-QAM transmission 


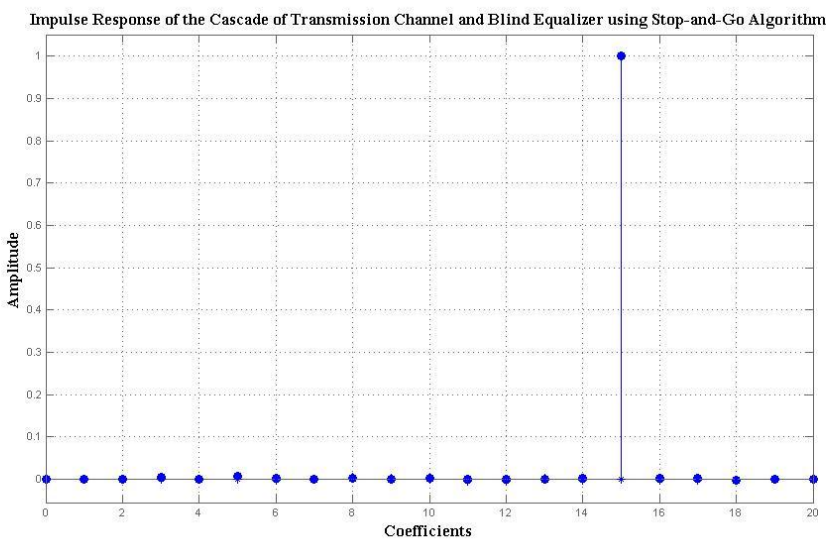

Fig.6 Impulse response of the cascade of transmission channel and the Stop-and-Go blind equalizer for 64QAM transmission

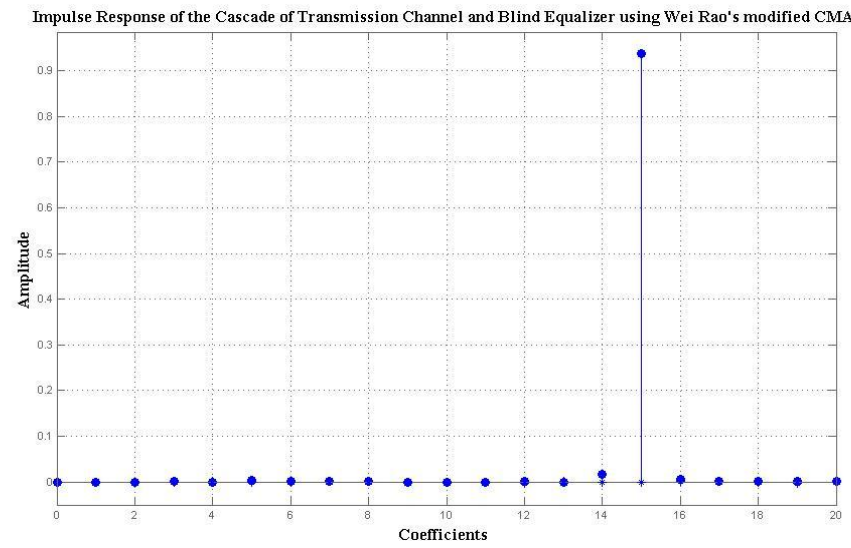

Fig.7 Impulse response of the cascade of transmission channel and the Wei Rao's modified CMA blind equalizer for 64-QAM transmission

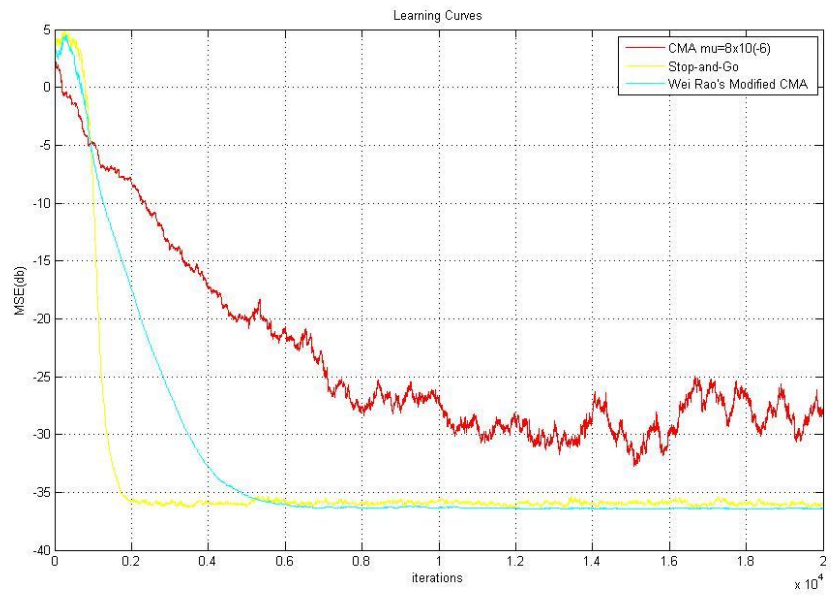

Fig.8 Learning Curves of CMA, Stop-and-Go and Wei Rao's modified CMA for 16-QAM Transmission 


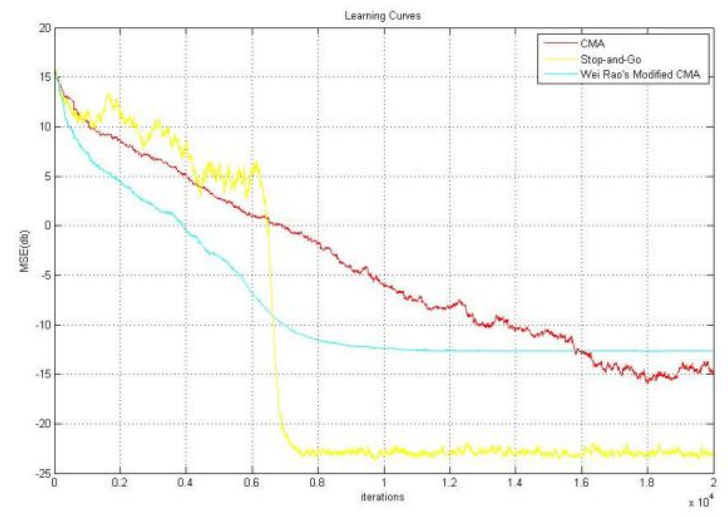

Fig.9 Learning Curves of CMA, Stop-and-Go and Wei Rao's modified CMA for 64-QAM Transmission

\section{Conclusion}

It is evident from figures 2-7 that all the three algorithms for both 16-QAM and 64-QAM transmissions produce a cascaded impulse response that is very similar to the ideal impulse response. Hence it could be said that the three algorithms successfully perform equalization. In blind equalization the speed of convergence is the chief performance determining factor of the algorithm. Low MSE results in high reduction of ISI. From figures 8-9 it is clear that for both 16-QAM and 64-QAM transmission Stop-and-Go algorithm has higher convergence rate followed by Wei Rao's modified CMA and CMA. By a simple modification in the weight updation process, the standard decision-directed algorithm can become very effective by giving better performance than the popular CMA in terms of convergence, MSE and residual error variance. By simple modification of the equalizer's output coordinates Wei Rao and his team modified CMA, and this algorithm exhibited better performance in terms of convergence, MSE and residual error variance than CMA, thus serving its purpose. CMA offers low convergence rate and high MSE besides large residual variance. Thus, it is obvious that from the obtained results Stop-and-Go would be the ideal choice over Wei Rao's modified CMA and CMA for blind equalization.

\section{References}

[1]. Jhon G.Proakis, Digital Communications,4th ed.,Mc Graw Hill,2001.

[2]. Ram Nishanth Vanka, S Balarama Murty, B Chandra Mouli, "Performance Comparison of Supervized and Unsupervized/Blind Equalization Algorithms for QAM Transmitted Constellations," International Conference on Signal Processing and Integrated Networks (SPIN), pp. 316-321, Feb.2014.

[3]. Y. Sato, "A method of self-recovering equalization for multilevel amplitude-modulation systems," IEEE Trans. Commun., vol.COM-23, pp. 679-682, June 1975.

[4]. D.Godard, "Self-recovering equalization and carrier tracking in two dimensional data," IEEE Trans. Communications, 1980, Vol.COM-28, pp.1867-1875.

[5]. G.Picchi and G. Prati, "Blind Equalization and carrier recovery using a stop-and-go decision directed algorithm,” IEEE Trans. Communications, vol.COM-35, Sept.1987.

[6]. Wei Rao, Jing-jing Lian and etc, "Simple Approach for Joint Blind Equalization and Order Detection Suitable for QAM Signal", 2009 IEEE International Workshop in Imaging System \& Techniques Proceedings, pp. 344- 347, May 11-12, 2009.

[7]. Haykin S, Adaptive Filter Theory, $4^{\text {th }}$ Ed, Pearson education, Inc., 2002

[8]. J.E. Mazo, “Analysis of decision-directed equalizer convergence,” Bell Syst.Tech. J., vol.59, pp. 1857-1876, Dec. 1980.

[9]. Wei Rao, Jing-jing Lian and etc, "A Simple Constant Modulus Algorithm for Blind Equalization suitable for 16-QAM signal", the $9^{\text {th }}$ conference on signal processing, vol. 2, pp.1963-1966, 2008. 